STUDIA ROMANICA POSNANIENSIA

UAM Vol. XLI/4 Poznań 2014

\title{
ALESSIO GIANNANTI
}

Università di Sassari

alessiogiannanti@libero.it

\section{LA SICILIA COME METAFORA: FEDERICO DE ROBERTO E TOMASI DI LAMPEDUSA}

\begin{abstract}
A b s t r a c t. Alessio Giannanti, La Sicilia come metafora: Federico De Roberto e Tomasi di Lampedusa [Sicily as metaphor: Federico De Roberto and Tomasi di Lampedusa], Studia Romanica Posnaniensia, Adam Mickiewicz University Press, Poznań, vol. XLI/4: 2014, pp. 47-60. ISBN 978-83-232-2791-5. ISSN 0137-2475. DOI: 10.7169/strop2014.414.005
\end{abstract}

The aim of this work, taking into account the last critical lesson of C.A. Madrignani, is to reflect upon how Sicily has become, through literature, a metaphor of the national political condition, and how it paradigmatically represents the inability of Italy to change. More precisely its purpose is to investigate the contribution to the construction of national identity of authors like F. De Roberto and G. Tommasi di Lampedusa, whose "anti-historical" works are characterized by a desecrating description (sometimes even polemical) of the unification process of Italy. Even this illusory contradiction is part of the dimension referred to by Madrignani as the Sicily effect on Italian literature. In Viceré and Imperio (and even in the war novellas) De Roberto bluntly depicts Italian history as a fierce fight for power, where human egoism prevails, which, according to a pessimistic philosophy, denies any affirmation of positive values.

Ke y w ord s: De Roberto, Lampedusa, Madrignani, history, pessimism

In questo intervento non intendo soltanto riflettere sulla dimensione perdurante di quello che è stato definito Carlo Alberto Madrignani l'Effetto Sicilia della letteratura italiana, ma cercherò anche d'intrecciare i temi di una ricerca più che quarantennale dello studioso con quelli che sono i miei piccoli passi sugli stessi sentieri. C'è un aspetto per me molto importante e che mi preme qui sottolineare- Se è vero che un maestro ci lascia non solo una metodologia di lavoro ma anche un modello didattico, ogni iniziativa che intenda omaggiare il magistero di Madrignani, dovrebbe tenere in considerazione questo aspetto: la sua era una didattica ostinatamente maieutica che quasi aboliva la lezione tradizionale (frontale e monologica) rivolta a dei ricettori più o meno passivi; nelle sue lezioni, attraverso un serrato corpo a corpo con i testi, si realizzava un continuo dialogo con gli studenti. Sono chiare le componenti civili, democratiche e progressiste di tale credo didattico, che in lui si manifestavano anche in un originale stile intellettuale e comunicativo. Nel momento in cui i suoi allievi sono stati chiamati ad un ultimo saluto, io e Giuseppe 
Lo Castro abbiamo fatto riferimento ad una frase del filologo italiano Giorgio Pasquali «i maestri vanno mangiati in salsa piccante», diventata celebre grazie al film di Pier Paolo Pasolini Uccellacci e uccellini. Non è certo una frase irrispettosa, ma ci serviva a restituire l'idea di un maestro che non vuole fare dei suoi allievi un clone, un'immagine a propria somiglianza, ma li sprona a superarlo, a fare qualcosa di diverso, in altre parole, ad esercitarsi continuamente nella ginnastica dell'intelletto e del pensiero critico.

L'ultimo libro di Madrignani, scritto quasi in limine mortis, ha come sottotitolo Genesi del romanzo moderno. Sono testimone del fatto che un altro sottotitolo era stato precedentemente pensato: Autobiografia di un'isola. Entrambi i sottotitoli sembrano sottintendere una domanda che è alla base di tutto il libro: «che cosa sarebbe la letteratura italiana moderna senza la Sicilia?» Se, poniamo caso, la Sicilia non fosse italiana; per esempio se le fosse capitato il destino che capitò a Malta e fosse stata annessa all'Impero Britannico? È ovviamente una provocazione, però serve a ribadire una semplice constatazione: non si capisce il romanzo moderno italiano se non si tiene conto della produzione di quella triade catanese che apre la strada alla modernità narrativa. I postcolonial studies hanno confermato il generale e fondamentale apporto delle provincie alla costruzione di un canone letterario nazionale, ciò è tanto più vero per la storia della cultura italiana che, come è noto, è fatta di frammentazione politica, ma anche linguistica e culturale. L'Effetto Sicilia ha oltre ad un suo specifico geografico-letterario anche uno politico e sembra che, in tutte le fasi storiche, le pagine più tristi della storia italiana, ma anche le ripartenze più felici, debbano incrociare i suoi destini con la Sicilia.

Il titolo del mio contributo riprende l'espressione sciasciana "la Sicilia come metafora politica", che è sottintesa nel discorso sull'Effetto Sicilia di Madrignani, e che a me qui serve per mettere in connessione, in un dittico che dichiaro subito un po’ sproporzionato, De Roberto e Tomasi di Lampedusa.

Il più grande peccato della Sicilia è stato ed è quello di non credere nelle idee, qui che le idee muovano il mondo non si è mai creduto. $\mathrm{Ci}$ sono naturalmente delle ragioni, di storia, di esperienze, però è questo che ha impedito sempre alla Sicilia di andare avanti, il credere che il mondo non potrà mai essere diverso da come è stato. Ora siccome questa mancanza di idee ormai si proietta su tutto il mondo, in questo senso per me la Sicilia ne è diventata la metafora (Sciascia, 1979) ${ }^{1}$.

Sono parole molto disilluse e amareggiate, che si collocano come alla fine di un percorso e ben esprimono quella mitografia che la Sicilia ha costruito su se stessa. Anche Madrignani, nell'elaborare i vari capitoli del suo libro, tiene presente questa interpretazione di Sciascia ed è come se studiasse a ritroso le diverse declinazioni di questo sentimento negli autori siciliani.

\footnotetext{
${ }^{1} \mathrm{Si}$ veda anche Intervista televisiva a Sciascia della RAI TV.
} 
Entro più nel merito del mio intervento raccontandovi un fatto di costume. Nel corso dei festeggiamenti per il $150^{\circ}$ anniversario dell'Unità d'Italia, ci si è interrogati sul contributo della letteratura alla formazione della nostra identità nazionale, anche in contesti non specialistici, tant'è che in molti hanno azzardato bollettini, graduatorie, classifiche di quali libri, o meglio, di quali opere letterarie fossero più significative e degne a commemorare il commemorabile. Con buona pace di Harold Bloom, quando si vuole sancire appartenenze ad ipotetici canoni, ogni elenco si rivela sempre insoddisfacente per inclusioni ed estromissioni assai discutibili; persino il super elenco di 150 libri, presentato al Salone del Libro di Torino del 2011, e che quindi si basava su una cifra non piccola, non è riuscito a fare eccezione, a contravvenire alla delusione (per non dire poi dell'elenco in 15 libri che risultava assolutamente inadeguato, anche ai fini del discorso che stiamo qui svolgendo).

Se è vero che l'Italia è stata per molti secoli un'idea letteraria, prima ancora che un'aspirazione geopolitica, e quindi un concreto riferimento a un modello linguistico e letterario, allora il ruolo svolto dalla letteratura nella "costruzione" della nazione è innegabile; a patto però di non intendere le opere letterarie soltanto come un propulsore di coscienze, una chiamata alle armi in favore degli ideali patriottici, ma anche, fuori da ogni dimensione propositiva, come uno specchio rovesciato degli umori e del clima politico e sociale del paese. È forse scontato dire che una tale prospettiva è al riparo da ogni tentazione destoricizzante e da quella religione del testo, la cui prassi quando degenerava, Madrignani chiamava "isolazionista", ma si fonda, al contrario, su un metodo di indagine storiografica e critico-letteraria che riconosce nella cultura il nutrimento di ogni linguaggio artistico e che ritiene $i$ fatti letterari non solo inscindibili dai fatti sociali, ma anche documenti utili a illuminare la storia.

In molti dei romanzi pubblicati dopo l'Unità possiamo rintracciare atteggiamenti critici (che tuttavia solo raramente sfociano nella vera e propria denuncia) verso la situazione politica, sociale e morale del nuovo stato. Il fulcro della vita politica, ovvero il Parlamento, viene spesso scelto come ambientazione delle storie, soddisfacendo in questo modo anche un desiderio d'informazione dei ceti medi, che ambivano ad essere resi partecipi dei cambiamenti in corso nella vita nazionale. L'interesse per la politica si fa così forte che viene anche consacrato un genere romanzesco ad hoc, il cosiddetto "romanzo parlamentare", che nella seconda metà dell'Ottocento ha visto cimentarsi alcuni dei più importanti narratori italiani (D’Annunzio, Fogazzaro, Serao, Guerrazzi, Rovetta, Del Balzo, Petruccelli della Gattina, oltre ovviamente a De Roberto $)^{2}$.

\footnotetext{
${ }^{2}$ Si veda l'antologia AA. VV. (1980): Rosso e nero a Montecitorio. Il romanzo parlamentare della nuova Italia (1861-1901), a cura di C.A. Madrignani, Firenze: Vallecchi. La parte introduttiva di questo saggio è stata ripresa e ampliata in Madrignani Carlo Alberto - Bertoncini Giancarlo (2001). Si veda anche il precedente Briganti (1972).
} 
Gli scrittori esprimono attraverso queste opere lo scontento degli intellettuali post-unitari per una Italia che non aveva saputo mantenere fede agli ideali che avevano animato la lotta irredentistica. Tra questi scrittori c'è senz'altro anche Federico De Roberto, che non solo è autore di uno dei più significativi romanzi (anti)parlamentari, ma la stessa trilogia della famiglia Uzeda-Francalanza potrebbe essere vista come la storia in controluce della nazione italiana, attraverso l'impietoso racconto della lotta crudele e degenerata di una famiglia siciliana per il mantenimento del potere $^{3}$. La posizione di De Roberto, che è rappresentante autorevole di questo risorgimentalismo critico, ha tuttavia qualche particolarità da tenere in considerazione. Innanzitutto dobbiamo pensare che le sue opere ebbero scarsissima circolazione in vita (è risaputo che il suo più importante romanzo non riuscì ad esaurire le duemila copie della prima edizione in quasi trent'anni); un fattore questo che è anche alla base di una valorizzazione critica assai tardiva. È quindi legittimo domandarsi quale effettivo contributo possa aver dato l'autore all'immaginario collettivo, al dibattito coevo su questi temi, visto che il disinteresse del pubblico era così conclamato (a discapito di un apprezzamento quasi generalizzato tra gli addetti ai lavori) che già ai primi del Novecento si iniziò a parlare di un «caso De Roberto» ${ }^{4}$.

Vi sono almeno tre condizioni che rendono particolarmente propizia un'esplorazione della sua opera in rapporto alla nuova identità nazionale: la prima è di natura anagrafica, nascendo nel 1861, egli è esponente della prima generazione di intellettuali postunitari, che formatasi nel mito del Risorgimento fu anche quella che forse scontò la delusione più grande; il secondo fattore ha a che fare con la provenienza culturale e geografica, ovvero essere parte di quella periferia privilegiata del romanzo moderno italiano che è costituita dalla Sicilia L'ultima condizione è relativa al suo profilo letterario: se da una parte De Roberto è intellettuale appartato, anticonformista e riluttante verso la politica, dall'altra, sposando l'oggettività naturalista (per quanto sottoposta ad una torsione sperimentale pre-novecentesca) e il realismo critico, utilizza un potentissimo strumento di analisi del mondo, che ha valenze preterintenzionali, ma non per questo meno politiche (Lukàcs avrebbe detto "non meno sincere").

Rispetto al rapporto di De Roberto con la nuova Italia, un primo riferimento (piuttosto battuto dalla bibliografia derobertiana) è appunto alla grande macchina

\footnotetext{
${ }^{3}$ Queste le prime edizioni della trilogia: De Roberto (1891), (1894) e (1929). Nel corso dell'intervento si citeranno i romanzi dal meridiano De Roberto (1998).

${ }^{4}$ «È conosciutissimo: e dovrebbe essere celebre. È molto apprezzato: e dovrebbe essere onorato come uno dei più forti ingegni d'Italia», è il commento che si può leggere nel profilo dedicato allo scrittore in De Frenzi (1904). Per la storia della critica derobertiana si vedano i capitoli «La ricezione e la storia della critica» e «Antologia della critica», in: Cavalli Pasini (1996: 97-206; 239-276) e il recente contributo di Castelli (2010).
} 
narrativa dei Viceré. Il capolavoro mai più eguagliato dallo scrittore sembra segnare una linea spartiacque dell'intera sua produzione, dopo il quale si registra una forte crisi e un esaurimento della vena creativa $\mathrm{E}$ tuttavia il romanzo esercitò un grande fascino (sebbene "a rilascio lento") sugli scrittori successivi, visto che esiste - come ha rilevato Spinazzola nel celebre saggio sul romanzo anti-storico - un filo rosso che unisce quest'opera ai Vecchi e i giovani di Pirandello e, appunto, al Gattopardo: tre romanzi in cui il racconto della parabola delle classi dirigenti siciliane diventa il tramite per un giudizio disincantato e severo sul processo di unificazione, alla cui base vi è la pressoché totale sfiducia nelle sorti progressive della storia ${ }^{5}$.

La storia della famiglia e della lotta per l'eredità della principessa Teresa, hanno per corollario il tema di una razza che combatte contro l'estinguersi delle proprie forze e un universo spietato dove non esiste solidarietà ma soltanto cattiveria, cinismo, opportunismo rampante, in cui si realizza uno stato incessante di guerra di tutti contro tutti, che non lascia tregua né serenità.

La prima parte del romanzo si svolge tra il 1855 e il 1861 e quindi comprende anche l'arrivo di Garibaldi in Sicilia. La Rivoluzione porta dapprima scompiglio nell'aristocrazia siciliana ma poi sono tutti pronti a salire sul carro del vincitore, compresi gli Uzeda Francalanza che si erano dimostrati contrari. Il tema politico è svolto attraverso la vicenda di Gaspare Uzeda duca d'Oragua, fratello del principe Giacomo e zio di Consalvo, che partito borbone e codino diventa tempestivamente liberale e democratico pur di poter cavalcare la Rivoluzione e trarne i maggiori benefici possibili. Sulle prime la famiglia Uzeda è indignata per questa frequentazione del Duca con i filo piemontesi, gente di rango inferiore ed esagitati che avevano soffiato sul fuoco del '48 predicando l'eresia democratica e anti-aristocratica. Tuttavia il Duca riuscirà, in seguito, a convincere i famigliari più avveduti che la politica è un'arte del raggiro e un inganno a danno dei più deboli, ma soprattutto che partecipare alla costruzione dell'Italia e alla cacciata dei Borboni era stato indispensabile ai Viceré per poter mantenere i privilegi del proprio rango. L'opportunismo e il trasformismo delle classi dirigenti siciliane realizza un immobilismo sociale che è immune da ogni reale trasformazione e impermeabile ai migliori intenti patriottici; una situazione che nel romanzo viene condensata nella parafrasi che il Duca d'Oragua fa della celebre frase attribuita a Massimo D'Azeglio: «Ora che l'Italia è fatta, dobbiamo fare gli affari nostri...». (De Roberto, 1998: 864).

Il personaggio di Gaspare Uzeda è portatore di una mediocrità priva di alcuna dignità e forse proprio per questo - pare sottintendere il narratore - è destinato a trionfare nella politica della nuova Italia. Il Duca d'Oragua - abile soltanto a fare i propri affari e incapace (fino al ridicolo) di parlare in pubblico - viene quindi eletto nel collegio di Catania alle prime elezioni sotto i Savoia grazie all'impegno dei

\footnotetext{
${ }^{5}$ Cfr. Spinazzola (1990).
} 
borghesi liberali, che vedono nelle sue nobili origini il segno di una maggior distinzione. Anche i patrioti sinceri vengono trattati dal narratore con la stessa fredda lucidità: non solo si dimostrano subalterni alla vecchia aristocrazia borbonica, ma il giovane idealista Benedetto Giulente è reso con impietosi tratti macchiettistici e caricaturali.

La grande storia, tuttavia, è come tenuta sullo sfondo: il Generale Garibaldi è ridotto a semplice comparsa e il focus rimane sempre sulle vicende della famiglia. Un tale ribaltamento delle proporzioni, in favore della cronaca famigliare e privata, costituisce un contrappunto simbolico di grande efficacia espressiva. Ad esempio, nella conclusione della prima parte, che rimane una delle pagine più famose dell'intero De Roberto, troviamo il parto di un feto mostruoso da parte di Chiara Uzeda che da tempo cerca, senza riuscirvi, di avere una progenie: l'evento si colloca proprio mentre, il duca D'Oragua viene eletto al parlamento, scendendo a patti con i liberali, e la sorella Lucrezia viene chiesta in sposa, con generale disapprovazione, dall'intraprendente avvocato Giulente. Il parto dell'abominio - o come sentenzia il narratore: «il prodotto più fresco della razza dei Viceré» (De Roberto, 1998: 694) - sancisce quindi sul piano allegorico la compromissione degli Uzeda con il nuovo regime dei Savoia, è il segnale inconfondibile della degenerazione patologica della stirpe, della natura irrimediabilmente teratogena del potere, ma forse è anche la rappresentazione più agghiacciante che sia stata fatta della nascita d'Italia.

Anche la seconda parte del romanzo si conclude con un'altra conversione, quella del monaco benedettino don Blasco, che dopo la presa di Roma mette da parte il suo clericalismo reazionario. Con la terza e conclusiva parte si arriva al 1882, quando viene sancita la definitiva ascesa politica del giovane Consalvo, che nel frattempo è diventato principe e sindaco di Catania. Particolarmente significativa è la descrizione della scena di massa che accoglie il comizio di Consalvo (a cui è dedicato un capitolo di Effetto Sicilia) per le elezioni a suffragio quasi universale. Siamo di fronte a un capolavoro di retorica politica e patriottarda: attraverso le capacità mimetiche del narratore si realizza uno stravolgimento parodistico che ci fa leggere tutta la demagogia e la sete di potere che anima il principe, disposto persino a farsi passare per progressista pur di raggiungere i suoi obiettivi. Nel discorso si scorge anche una tramatura sottile con frequenti slittamenti di significato: si passa dagli interessi particolari e localistici dei siciliani all'evocazione pomposa di una dimensione ormai pienamente nazionale (la «gran patria comune») ma poi si torna indietro seguendo il percorso inverso, passando dal generale al particolare si realizza un effetto smascheratamente corrosivo (De Roberto, 1998: 1085). Si veda la seguente formulazione in climax discendente:

Il pensiero della patria nostra è quest'Italia che il pensiero di Dante divinò, e che i nostri padri ci diedero a costo di sangue (Vivissimi applausi). La nostra patria è anche quest'isola benedetta dal sole, dov'ebbe culla il dolce stil novo e donde partirono le più gloriose iniziative 
(Nuovi applausi). La nostra patria è finalmente questa cara e bella città dove noi tutti formiamo come una sola famiglia (Acclamazioni). Dicesi che i deputati rappresentano la Nazione e non i singoli collegi. Ma in che consistono gl'interessi nazionali se non nella somma degli interessi locali? (Benissimo, applausi) (De Roberto, 1998: 1090-1092).

La voce narrante sembra come sdegnarsi e porre un filtro di ironia sottile e quasi impercettibile nelle notazioni stenografiche e nelle descrizioni icastiche delle reazioni della platea.

Le ultime pagine del romanzo coincidono con l'elezione al parlamento del giovane Uzeda e con il congedo, prima di partire per Roma, dalla austera matriarca Donna Ferdinanda, ancora legata al mondo borbonico e la più rancorosa della famiglia verso i cambiamenti. Consalvo, nel rassicurare la zia sul fatto che i Viceré con la nascita dell'Italia non hanno perso il loro antico prestigio, esprime quella filosofia negativa sulle sorti umane che è alla base dell'intero romanzo: «La storia è una monotona ripetizione, gli uomini sono stati, sono e saranno sempre gli stessi» (De Roberto, 1998: 1100).

Questa filosofia negativa sul piano della storia era già stata descritta sul terreno delle relazioni amorose attraverso la perlustrazione monologante di Teresa Uzeda, nel romanzo L'Illusione (1891), e in seguito animerà la trattatistica amorosa, che si inaugura con gli apologhi La morte dell'amore (1892) e che trova uno degli esiti più significativi nel ponderoso volume L'amore: fisiologia, psicologia, morale (1895) ${ }^{6}$. Pochi anni dopo l'uscita dei Viceré, De Roberto si cimenterà nello studio su Leopardi (1898a $)^{7}$, a testimonianza di un rispecchiamento nella sua filosofia pessimista e di un'inquietudine intellettuale che se a livello personale ha - per ammissione dello stesso autore - delle complicazioni di carattere nevrotico (la «follia del dubbio», come la chiama egli stesso) ${ }^{8}$, dal punto di vista del dibattito letterario è invece da ricondurre alla coeva crisi del Naturalismo. Il pessimismo e lo scetticismo radicale che prendono, con gli anni, sempre più piede nelle riflessioni e nelle opere di De Roberto sono il risultato di un esercizio continuo di scepsi che lo porta a mettere a dura prova la validità degli strumenti di analisi razionale: mettendo tutto in dubbio si erode dall'interno ogni ortodossia positivista.

Un crollo totale di ogni fiducia nel progresso, nonché negli ideali Risorgimentali, è quello che si legge nell'Imperio, il «libro terribile» che De Roberto non riuscì

${ }^{6}$ La trattatistica amorosa derobertiana è costituita dalle seguenti opere: De Roberto (1892): La morte dell'amore, Napoli: Pierro, poi ripubblicato postumo con l'aggiunta di tre capitoli (Milano, Maia, 1928); De Roberto (1895): L'amore. Fisiologia, psicologia, morale, Milano: GalliChiesa-Guidani; De Roberto (1898b): Gli amori, Milano: Galli-Chiesa-Guidani (che ingloba La morte dell'amore); De Roberto Federico (1898c): Una pagina della storia dell'amore, Milano: Treves; De Roberto (1913): Le donne, i cavalier', Milano: Treves.

${ }^{7}$ De Roberto (1898a).

${ }^{8}$ Tale espressione, che è ripresa dalla scuola psichiatrica francese, si legge in una lettera del 23 dicembre 1916 al capocomico Virgilio Talli, in Lopez Sabarino (1931). 
mai a portare a termine - pur lavorandovi dal 1893 al 1896 e poi a fasi alterne fino almeno al 1910 - e che uscì postumo e incompleto nel $1929^{9}$. Il romanzo - che sempre secondo l'autore avrebbe dovuto avere «l'effetto d'una bomba» sulle coscienze degli italiani - prosegue la storia raccontata nei Viceré con l'arrivo di Consalvo Uzeda a Roma e il suo insediamento al parlamento. Con questa opera De Roberto partecipa all'ondata di romanzi parlamentari (più spesso anti-parlamentari) degli anni Novanta, che nacquero anche a seguito dell'indignazione per lo scandalo della Banca Romana.

Seguendo la storia di Consalvo ci inoltriamo in una Roma che, oltre ad essere la capitale della mondanità, è soprattutto il cuore della vita politica. Sin dalle prime pagine, la società capitolina è presentata come un mondo arido, regno dell'intrallazzo, dove tutti tramano per poterne ricavare un vantaggio personale, non diversamente dalla smania di ricchezza che muoveva gli Uzeda. La differenza è che nel Parlamento e nei salotti romani quella stessa brama si nasconde dietro i modi cortesi e le affettazioni; un'ipocrisia che risulta indigesta persino allo stesso Consalvo. E così, inizialmente, il principe è angosciato dal fatto che, se i Viceré erano idolatrati a Catania, a Roma non hanno lo stesso potere; pertanto dedica tutto se stesso, con il cinismo e l'avidità che gli appartengono, alla conquista di una qualche visibilità; fino a quando - anche grazie a coincidenze fortunate (ovvero è vittima di un attentato fallito, dopo suo discorso anti-socialista) - rivestirà la carica di Ministro degli Interni.

Alla vicenda di Consalvo, e alla sua ossessione per il successo, viene affiancata e, anzi, contrapposta la figura di Federico Ranaldi, un giovane intellettuale campano che - nato significativamente nel 1860, nel giorno della liberazione di Napoli appartiene a quella prima generazione di italiani che si era infatuata del mito risorgimentale e che vedeva nell'Unità d'Italia il compimento dei più nobili ideali. Federico facendo il giornalista, e in particolare il notista politico, per il quotidiano «Cronaca», diventa collaboratore del deputato Uzeda; ma, proprio attraverso il suo lavoro, viene presto a conoscenza della vera natura di questo mondo e della mediocrità e opportunismo che regna tra i rappresentati del Parlamento.

L'esperienza romana porta Federico ad un progressivo logoramento della fiducia nella nuova Italia e nei valori che lo avevano animato nella più fervida gioventù, fino al punto di cadere in un baratro di sconforto: egli si sente ormai complice dei giochi subdoli e degli imbrogli perpetrati dai politici e dal giornale. Nell'ultimo capitolo passiamo bruscamente (ma più che una scelta narrativa è un effetto

\footnotetext{
${ }^{9}$ Le fasi redazionali del romanzo, compresa l'ipotetica ripresa dei lavori intorno al 19091910, in occasione del soggiorno romano, sono state ricostruite attraverso il carteggio con la madre: De Roberto (1978). Tuttavia il coinvolgimento emotivo presente in questi documenti e il particolare rapporto tra lo scrittore e la madre rendono le dichiarazioni dello De Roberto tutt'altro che attestazioni, prove filologiche inconfutabili, come ha argomentato Di Grado (1998).
} 
dell'incompiutezza dell'opera) dai primi anni Ottanta al 1900, quando Ranaldi è ormai quarantenne. Lo scenario è apocalittico: abbandonata Roma, egli è tornato a Salerno dai genitori, in uno stato di disperazione che lo porta a propositi suicidi e ad una forma di radicale pessimismo che sfocia nel nichilismo più estremo. Nel patriota è ormai svilita ogni speranza, egli non crede più in nulla, adesso è convinto che la felicità sia sempre chimerica, così come ogni fiducia sia destinata a rivelarsi illusoria, e il Bene venga continuamente sopraffatto dal Male, che «dura, invariato, eterno, inesorabile» (De Roberto, 1998: 1352). L'unica consolazione possibile per l'uomo, la sola possibilità di una cessazione del dolore, è costituita dalla morte. Le pagine, che De Roberto dedica alla visione che Ranaldi ha del mondo e della vita come contagio e malattia (un enorme tumore che non può che condurre alla necrosi), raggiungono una forza espressiva e una qualità stilistica sopraffine, tra le prove più alte dello scrittore. La filosofia negativa della storia e la mancanza di fiducia nel Progresso, che erano già presenti nei Viceré, adesso vengono spinte da Ranaldi ben oltre: egli arriva a profetizzare, sulla scia di alcuni attentati anarchici, la comparsa di una setta di fanatici, detti «biofobi» e «geoclasti», che faranno esplodere il mondo e con esso il genere umano, perché questo rimane l'unico modo per poter liberare l'uomo dal Male (De Roberto, 1998: 1373-1376). Come è stato notato, non siamo lontani dalla palingenesi del capitolo finale della Coscienza di Zeno e, soprattutto, De Roberto con questo ultimo capitolo ci restituisce una lettura, tra le più radicalmente pessimiste, di quella crisi fin de siècle che costituisce il tramite per una nuova e più moderna coscienza. Tuttavia il narratore non sostiene fino in fondo l'eccesso stilistico ed ideologico, e in extremis il personaggio compie una rotazione consolatoria da feuilleton: la giovanissima Anna riesce ad allontanare Ranaldi dai propositi suicidi, attraverso una richiesta di matrimonio che viene, persino, suggellata dalla madre del futuro sposo (l'incompletezza dell'opera e la brusca accelerazione del finale non lasciano intendere se alla riconciliazione con le leggi della famiglia corrisponda anche un ritrovamento da parte di Federico della fiducia nei valori patriottici).

La violenza estrema dell'ultimo capitolo e l'amarezza al limite del psicopatologico provata da Ranaldi sono da mettere in relazione sia ad un riflesso autobiografico della condizione di prostrazione psicofisica che accompagnerà De Roberto fino agli ultimi suoi giorni, sia alla delusione per la perdita di significato dei valori risorgimentali che investì la generazione dello scrittore; e che in lui trova una declinazione ancor più radicalmente pessimista. Secondo Carlo Alberto Madrignani già nei Viceré si può leggere «un ripensamento in forma artistica della storia politica dell'Italia moderna» sebbene sia estranea all'autore una piena volontà di polemica politica (Madrignani, 1998: XXXII). Alla base vi è l'idea della Sicilia come metafora politica, in cui «la sua separatezza, il suo isolamento ed insieme la sua capacità di essere la controparte critica e lucida nei confronti dell'ufficialità continentale» 
(Madrignani, 1998: XXXIII) sono i tratti peculiari. Con L'imperio il discorso si fa più esplicito, la condanna più dura: il romanzo «è l'atto di sfida più argomentato, esplicito e brutale con cui un intellettuale del meridione ha sancito il distacco del sud dal nord ed ha fatto luce sulla corruzione molecolare e sistematica, organica ad ogni forma di potere» ${ }^{10}$. In questo senso, possiamo intendere De Roberto come un intellettuale critico, addirittura di opposizione: non certo perché egli si iscrive ad un programma politico alternativo (tra l'altro le istanze progressiste non sembrano scaldargli il cuore) ma perché il suo metodo di indagine e di rappresentazione del mondo, perché la forza demitizzante del suo realismo, ci hanno restituito una storia dell'Italia, totalmente irriducibile alla storiografia e alle ideologie ufficiali del suo tempo. De Roberto piuttosto che celebrare i supposti fasti della storia nazionale, ci offre un'immagine dell'unificazione che sembra quasi ridursi ad un episodio di trasformismo, di camaleontismo delle classi dirigenti.

Esiste anche una prospettiva diversa per guardare il rapporto dello scrittore con il Risorgimento, ovvero con l'identità e la storia italiana. Prendiamo l'«ultimo De Roberto» e in particolare quella fase in cui lo scrittore, ormai isolato nel rifugio catanese e a un passo dal definitivo ritiro, sembra come ritrovare una nuova lena artistica. L'adesione dello scrittore al nazionalismo di guerra è un ottimo banco di prova per verificare il suo atteggiamento verso la materia italiana (del resto secondo la propaganda irredentista la Prima Guerra Mondiale era una prosecuzione dello stesso Risorgimento). De Roberto non smentisce il suo profilo di scrittore di una complessità non priva di contraddizioni e dalla «eccezionale e sfuggente politicità» (Madrignani, 1998: XXXVII): se gli scritti saggistici (1914-1918), che accompagnano gli eventi bellici, attestano una lineare intenzione di propaganda bellica, le successive opere narrative (le cosiddette "novelle di guerra", 1918-1923) presentano elementi ambivalenti che, in taluni casi, contraddicono i propositi propagandistici: in un caso, nella novella La paura (1923), assistiamo addirittura ad un ribaltamento di significato, siamo in presenza di una spietata - per quanto forse non del tutto volontaria - denuncia della follia della guerra e della vacuità di ogni sacrificio in nome della "Patria". Anche altre novelle introducono un tarlo nella rappresentazione ufficiale degli eventi, poiché il metodo documentario e l'impegno realista di De Roberto non ammette limitazioni né autocensure. Vi è quindi un ritorno alla forza desacralizzante del suo realismo (fra l'altro con uno uso del dialetto che ha una

\footnotetext{
${ }^{10}$ Madrignani parla di «romanzo politico sui generis» per sottolineare la dimensione non programmatica delle critiche politiche contenute nelle opere derobertiane: «Di contro alla letteratura maggioritaria, tutta tesa a rivendicare la grandezza delle lotte risorgimentali e le novità della giovane politica dello stato sabaudo, De Roberto, senza proporsi nessun piano politico alternativo consapevole, scrive un'opera di intonazione antirisorgimentale, nel senso che non solo ogni tono apologetico viene respinto, ma vi si insinua uno spirito di corrosiva demistificazione» (Madrignani, 1998: XXXVI-XXXVII).
} 
matrice sperimentale di inedita efficacia) e ritroviamo la repulsa dell'autore nei confronti di qualsiasi potere costituito, che in lui è quasi sempre un rifiuto prepolitico, più determinato dal suo pessimismo misantropo che non da una critica ragionata, ma, come si è detto, non per questo ha minore efficacia di denuncia.

Riprendendo adesso il dubbio avanzato nella premessa al discorso sul rapporto tra letteratura e costruzione di una coscienza nazionale, potremmo chiederci: una letteratura che è portatrice di una visione così negativa della storia, e conseguentemente delle storie patrie, e uno scrittore che potremmo definire un italiano scettico - poiché continuamente sottopone a dura prova ogni mito unitario, ogni sentimento di concordia - possono contribuire alla definizione di una identità nazionale, italiana? La riposta a tale quesito - al saldo dalle banalizzazioni che promuovono gli elenchi ad uso giornalistico - è ovvia, ma nell'inevitabile euforia del clima celebrativo, si è forse rischiato di fare passare un'immagine necessariamente conciliante della letteratura verso il Risorgimento e non tenere nella dovuta considerazione una produzione, un filone (non esclusivamente meridionale o meridionalista) che intrattiene con quei fatti un atteggiamento scettico se non propriamente critico, di certo non pacificato.

Il discorso su Giuseppe Tomasi di Lampedusa non può non tenere conto della tradizione siciliana e del "precedente" di De Roberto: sicuramente Madrignani era convinto di questa derivazione. A Pisa, la città in cui ci siamo formati, abbiamo avuto la fortuna di poter seguire le lezioni di un grande teorico della letteratura: Francesco Orlando (1934-2010) fondatore di quella che è nota come la «teoria freudiana» della letteratura. Il giovane Orlando, un liceale proveniente dalla borghesia palermitana, era stato uno degli allievi di Tomasi di Lampedusa, e aveva materialmente battuto a macchina il dattiloscritto del Gattopardo. La storia del rapporto con lo scrittore di Orlando e dell'altro allievo, il musicologo Gioacchino Lanza Tomasi, è stata raccontata, sebbene in forma romanzata, nel film Il manoscritto del principe del 2000, regia di Roberto Andò.

Verso la fine degli anni Novanta, Francesco Orlando scrisse un saggio che si intitola L'intimità e la storia. Lettura del "Gattopardo" (1998). È un libro molto bello, perché dallo spunto iniziale, ovvero la partecipazione diretta alla realizzazione di questo romanzo, si passa ad un'analisi testuale rigorosa, in cui vengono messi in mostra gli strumenti raffinati del teorico e del comparatista. Che cosa c'entra in questa vicenda Madrignani? Le posizioni sull'opera dei due studiosi sono distanti e anzi contrapposte: da una parte Madrignani, che è uno dei massimi esperti di letteratura siciliana e legge il romanzo in continuità con il retroterra insulare, dall'altra Orlando, la cui lettura tende a riconnettere il romanzo a una radice europea (ad esempio La Chartreuse de Parme di Stendhal) e allo stesso tempo rifiuta la connessione con la letteratura regionale e siciliana. Per Orlando la Sicilia di questo romanzo è il paradigma della periferia, ovvero di qualsiasi periferia nel mondo, in 
cui ogni lettore può immedesimarsi (e ciò spiegherebbe anche il successo internazionale). È una lettura legittima ma che non poteva trovare d'accordo Carlo Alberto Madrignani. Qui capita agli studenti pisani di quegli anni un'opportunità che non dimenticheranno facilmente: nello stesso anno accademico i due studiosi tengono, con queste due diverse impostazioni, un corso incrociato sul romanzo. Mi pare che nessuno abbia mai scritto su questa vicenda, ma sarebbe interessante raccontarla anche come esperienza didattica: certo su molti aspetti le due letture convergono, tuttavia rimane la contrapposizione principale nell'interpretazione del valore del rapporto con la tradizione siciliana, anche se vista come negazione e stravolgimento. Madrignani farà confluire questa lettura seminariale in un articolo contenuto in un volume in onore di Umberto Carpi, dal titolo Il romanzo della «terrificante insularità», poi ricollocato come un capitolo dell'ultimo libro, in cui il Gattopardo è ricollegato all'effetto Sicilia.

È noto che il Gattopardo fu un'opera in grado di dividere da subito (celebre è la storia del rifiuto di Vittorini); ad esempio il giudizio di Franco Fortini è molto severo, la considerava un'opera reazionaria non antistorica (come dice Spinazzola) ma "contro la storia", perché a suo parere la falsificava e anche Calvino trattava quest'opera con una certa sufficienza. Invece un altro grande intellettuale della sinistra italiana, Luchino Visconti ha amato questo romanzo e con il suo film ne ha amplificato il successo.

È interessante guardare questo romanzo dalla prospettiva dell'Effetto Sicilia e constatare che quella automitografia, fondata sulla diversità e sull'immobilità dei siciliani, trova qui una delle più durature rappresentazioni. Si potrebbe dire che il Consalvo delle ultime pagine dei Viceré e don Fabrizio sono, per certi versi, in continuità, e tuttavia prevalgono gli elementi di differenziazione: per esempio in De Roberto non c'è partecipazione nei confronti dei propri personaggi, come per Tomasi di Lampedusa rispetto a don Fabrizio, che ci è presentato come un vecchio e ormai stanco nobile, giunto alla fine della sua vita (centrale è la riflessione sulla morte), che decide, quasi eroicamente, di lasciare il potere perché non lo interessa più. Madrignani sottolinea anche un altro aspetto: la metaforizzazione della Sicilia nel Gatttopardo assume una dimensione più didascalica e insieme più esplicita, è come se si tagliassero i ponti con quella essenzialità e quell'obliquità, che erano alla base della verità scomoda di Verga. Una metaforizzazione che nel Gattopardo tende a dematerializzare e a nascondere le condizioni di vita dei Siciliani, restituendo una versione compensativa e confortevole della verità verghiana il romanzo mette in scena soprattutto la crisi metastorica di un individuo. A mio parere, un altro elemento che distingue Tomasi da Lampedusa da un Verga o da un De Roberto è che il loro fatalismo non è mai vittimistico, nel Gattopardo invece questa dimensione è amplificata, e sviluppata insieme al tema della bellezza terribile e abbagliante, a cui si ricollega l'altro mito dell'Isola del sole (come si intitolava un 
libello di Luigi Capuana), ma anche qui è declinato in una prospettiva edonistica sconosciuta agli scrittori veristi.

Il Gattopardo nell'impianto interpretativo di Madrignani è una tappa fondamentale per definire quell'Effetto Sicilia, che nel libro si fa partire dalle origini (Verga e ancor prima Navarro della Miraglia) fino alle manifestazioni contemporanee: Vincenzo Consolo e Andrea Camilleri, che sono i principali eredi in una stagione definita, significativamente, "dopo Sciascia".

Ho voluto citare la lettura incrociata di due maestri che non ci sono più, perché penso che questo episodio in termini didattici e metodologici ci insegni qualcosa di utile: penso soprattutto a quelle e quegli studenti, e in generale ai giovani in formazione che si interrogano quotidianamente su quello che leggono. Mi sembra un caso utile a dimostrare quante opzioni, in fatto di interpretazioni dei testi, abbiamo davanti e come nel futuro sia per loro indispensabile costruire una cassetta degli attrezzi - che è la cassetta del metodo di ricerca, dell'analisi critica dei testi - che sia la più fornita possibile; e saper raccogliere spunti da letture anche molto diverse di uno stesso testo, in altre parole tentare sempre di mettere in discussioni verità assodate che paiono inconfutabili. Questo è il lascito più profondo della lezione di Carlo A. Madrignani, con cui ribadire l'opportunità, e direi la necessità, che il ricordo di ogni grande magistero non sia un punto di approdo, ma sia sempre un punto di ripartenza, per nuove possibili interpretazioni.

\section{BIBLIOGRAFIA}

AA. VV. (1980): Rosso e nero a Montecitorio. Il romanzo parlamentare della nuova Italia (18611901), a cura di C.A. Madrignani. Firenze: Vallecchi.

BRIGANTI, Alessandra (1972): Il parlamento nel romanzo italiano del secondo Ottocento. Firenze: Le Monnier.

Castelli, Rosario (2010): Il punto su Federico De Roberto. Per una storia delle opere e della critica. Acireale-Roma: Bonanno.

Cavalli Pasini, Annamaria (1996): De Roberto. Palermo: Palumbo.

De FrenzI, Giulio [pseud. di Luigi Federzoni] (1904): «Federigo De Roberto», in: Candidati all'immortalità. Bologna: Zanichelli.

De Roberto, Federico (1891): L'illusione. Milano: Galli-Chiesa-Guindani.

De Roberto, Federico (1892): La morte dell'amore. Napoli: Pierro.

De RoBerto, Federico (1894): I Viceré. Milano: Galli-Chiesa-Guindani.

De Roberto, Federico (1895): L'amore. Fisiologia, psicologia, morale. Milano: Galli-ChiesaGuidani.

De Roberto, Federico (1898a): Leopardi. Milano: Treves.

De Roberto, Federico (1898b): Gli amori. Milano: Galli-Chiesa-Guidani.

De Roberto, Federico (1898c): Una pagina della storia dell'amore. Milano: Treves.

De Roberto, Federico (1913): Le donne, i cavalier'. Milano: Treves.

De Roberto, Federico (1928): La morte dell'amore. Milano: Maia.

De Roberto, Federico (1929): L'imperio. Milano: Mondadori. 
De Roberto, Federico (1978): Lettere a donna Marianna degli Asmundo, a cura di S. Zappulla Muscarà. Catania: Trincale.

De Roberto, Federico (1998): Romanzi, novelle e saggi, a cura di C.A. Madrignani. Milano: Mondadori [prima edizione 1984].

Di GRADO, Antonio (1998): La vita, le carte, i turbamenti di Federico De Roberto, gentiluomo. Catania: Fondazione Verga.

Sabarino, Lopez (1931): Dal carteggio di Virgilio Talli, raccolto da E. Roggero. Milano: Treves.

MAdrignani, Carlo Alberto; BerTONCINI, Giancarlo (2001): „Il Parlamento nel romanzo italiano”, in: Storia d'Italia. Annali 17. Il Parlamento, a cura di L. Violante con la collaborazione di Francesca Piazza. Torino: Einaudi, 931-966.

MAdRignani, Carlo Alberto (1972): Illusione e realtà nell'opera di Federico De Roberto. Saggio su ideologia e tecniche narrative. Bari: De Donato.

MADRIGNANI, Carlo Alberto (1998): „Introduzione”, in: De Roberto Federico, Romanzi, novelle e saggi, a cura di C.A. Madrignani. Milano: Mondadori [prima edizione 1984].

Madrignani, Carlo Alberto (2007): Effetto Sicilia. Genesi del romanzo moderno. Macerata: Quodlibet.

ORLANDO, Francesco (1998): L'intimità e la storia. Lettura del "Gattopardo". Torino: Einaudi.

SCIASCIA, Leonardo (1979): La Sicilia come metafora, intervista di Marcelle Padovani. Milano: Mondadori.

Spinazzola, Vittorio (1990): Il romanzo antistorico. Roma: Editori Riuniti.

TOMASI Di LAMPEDUSA, Giuseppe (1958): Il Gattopardo. Milano: Feltrinelli. 